\title{
Acknowledgments
}

The Editor and Associate Editors gratefully acknowledge the following principal reviewers who reviewed manuscripts for Developmental Psychology's 2020 volume.
Linda Camras
Tutrang Nguyen
Michael Willoughby
Brett Laursen
Bart Soenens

The Editor and Associate Editors gratefully acknowledge the following ad hoc reviewers who reviewed manuscripts for Developmental Psychology's 2020 volume.

\begin{tabular}{|c|c|c|c|}
\hline Monika Abels & Doris Baker & Clancy Blair & Adriana G. Bus \\
\hline Aktan Acar & Marian J. Bakermans- & Peter R. Blake & Aaron Buss \\
\hline Byron Adams & Kranenburg & Erik Blaser & Sheretta T. Butler-Barnes \\
\hline Ryan Adams & Aida B. Balsano & Matthias Bluemke & David Buttelmann \\
\hline Menatalla Ads & Meeta Banerjee & Myrthe G. B. M. Boekhorst & Nicholas Buttrick \\
\hline Narges Afshordi & Colin Bannard & Gunilla Bohlin & \\
\hline Charlotte Agger & Miguel Barbosa & Natalie Bohlmann & Marie Camerota \\
\hline Richard E. Ahl & Ted Barker & Manuel Bohn & Valerie Camos \\
\hline Johan Ahlen & David Barner & Marie-Aude Boislard & Joseph J. Campos \\
\hline Sammy Ahmed & Marcia Barnes & Michel Boivin & Elizabeth Canning \\
\hline Lydia HaRim Ahn & Andrew S. Baron & Jordan A. Booker & Ryan Cannistraci $^{*}$ \\
\hline Lieselotte Ahnert & Alicia Barreiro & Amy Booth & Deborah M. Capaldi \\
\hline Kristine J. Ajrouch & Chris Barry & Corinne Bower & Serge Caparos \\
\hline Nazan Aksan & Ryan A. Barry-Anwar & Brittany P. Boyer & Simona Caravita \\
\hline Stephanie Al Otaiba & Igor Bascandziev & Ty W. Boyer & Fiorella L. Carlos Chavez \\
\hline Jamie Albright & John Bates & Barbara Braams & Stephanie M. Carlson \\
\hline Nicole Alea & Laurie Bayet & Robert H. Bradley & Nicola Carone \\
\hline Lenneke Alink & Benjamin L. Bayly & David William Braithwaite & Robert Carr \\
\hline Jennifer Allen & Anne Beatty-Martinez & Neil Bramley & Barbara Carretti \\
\hline Joseph Allen & Sarah R. Beck & Annie Brandes-Aitken & Jamee Carroll* \\
\hline Lauren Alvis & Michael Becker & Naemi Brandt* & Alexandra Carstensen \\
\hline Danming An & Marjorie Beeghly & Markus Brauer & Marianella Casasola \\
\hline Jim Anderson & Kazuko Y. Behrens & Elizabeth Brey & Michele Cascardi \\
\hline Kirsten Anderson* & Mona Bekkhus & Alexis Brieant ${ }^{*}$ & Katherine Casey* \\
\hline Nina Anderson & Martha Ann Bell & Thomas M. Brinthaupt & Marisa Casillas \\
\hline Riana Anderson & Daniel Belsky & Natalie Brito* & Laurence Casini \\
\hline Vicki Anderson & Viridiana Benitez & Aerika Brittian Loyd & Thomas Castelain \\
\hline Katherine Andrews* & Aprile D. Benner & Garvin Brod & Luigi Castelli \\
\hline Arya Ansari & David Bennett & Christina A. Brook & Anne Castles \\
\hline Toni C. Antonucci & Deon Benton & Rechele Brooks & Dina Castro \\
\hline Joanne Arciuli & Christian Berger & Alleyne Broomell & Nichol Castro \\
\hline Natalia Arias-Trejo & Christi Bergin & Holly Brophy-Herb & Susana Castro-Kemp \\
\hline Brian E. Armenta & Christina Bergmann & Patricia E. Brosseau-Liard & Timothy A. Cavell \\
\hline Laura Marie Armstrong & Laura E. Berk & Alexander S. Browman & Pablo Celhay \\
\hline Inbal Arnon & Cady Berkel & Christia Spears Brown & Natasha Chaku \\
\hline Maria Arredondo & Marvin W. Berkowitz & Geoffrey L. Brown & Lisa Chalik \\
\hline William Arsenio & Lisa Berlin & Sarah Brown & Craig Chambers \\
\hline Gisa Aschersleben & Annie Bernier & Laura E. Brumariu & Cheri Chan \\
\hline Mark H. Ashcraft & Daniel Berry & Eddie Brummelman & Christian S. Chan \\
\hline Eva Asselmann & Kelly Carmelita Berthiaume & Janina Larissa Bühler & Purificación Checa \\
\hline Olivia E. Atherton & Sara R. Berzenski & William M. Bukowski & David Chen* \\
\hline Allison Atteberry & John R. Best & Rebecca Bulotsky-Shearer & Eva E. Chen \\
\hline Danielle Augustine* & Tilmann Betsch & Marine Buon & Kuan-Hua Chen \\
\hline Mairin Augustine & Lin Bian & Margaret (Peg) R. Burchinal & Si Chen \\
\hline \multirow[t]{2}{*}{ Ziada Ayorech } & Ann E. Bigelow & Rebecca Burdayron* & Xinyin Chen \\
\hline & Rebecca S. Bigler & Julien S. Bureau & Yan Chen \\
\hline Farzaneh Badinlou & Patricia Bijttebier & Amanda R. Burkholder & Yi-Ling Cheng \\
\hline Catherine L. Bagwell & Asamenew Demessie Bireda & Kaitlyn Burnell & Dana L. Chesney \\
\hline Lorraine E. Bahrick & Kaj Bjorkqvist & Melissa Burnham & Cecilia Cheung \\
\hline J. Michael Bailey & Maureen Black & Anthony L. Burrow & Coralie Chevallier \\
\hline Bruce L. Baker & Jennifer Blackford* & Keith Burt & Jane B. Childers \\
\hline
\end{tabular}


Daniel Ewon Choe

Soonja Choi

Youjung Choi

Sophia Choukas-Bradley

Angela Chow

Kirby Chow

Sharon L. Christ

Georgia Chronaki

Joanne M. Chung

Elvira Cicognani

Andrei Cimpian

Paul T. Cirino

Andrew Clark

Caron Clark

Kirsty Clark

Bo Cleveland

Alexandra Cohen

Dale Cohen

Joseph Cohen

Shana Cohen

Rebekah Levine Coley

Andrew M. Colman

Katherine J. Conger

Bradley T. Conner

Kelly Conover

Clare Conry-Murray

Mariah M. Contreras

Andrew Conway

Caylee Cook

Elizabeth C. Coppola*

Daniela Corbetta

Cynthia Core

Katherine Corker

Rodolfo Cortes Barragan

Kai S. Cortina

William Coventry

Nelson Cowan

Philip A. Cowan

Jason Cowell

Nadia Craddock

Lucy Cragg

Ali Crandall

Sarah C. Creel

Dean Cristol

Sheila Crowell

E. Mark Cummings

Jenna Cummings**

Stephanie M. Curenton

Carolyn Cutrona

Lauren Cycyk

Christopher Daddis

Mark Dadds

Or Dagan*

Heather Daly

Fabrice Damon

Elizabeth Daniels

Linda Darling-Hammond

Shai Davidai

Telli Davoodi

Molly Dawes

Carina de Klerk

Jill G. de Villiers

Pascal R. Deboeck
Kathryn A. Degnan

Juliane Degner

Jasmine M. DeJesus

Dawn DeLay

Sarah DeMartini

Anne-Sophie Denault

Jaap J. A. Denissen

Joanne Deocampo

Paul Deutchman*

Rory T. Devine

Casey Dexter

Elisa Di Giorgio

Anjolii Diaz

Anthony Steven Dick

David Dickinson

Matthew Diemer

Julia Dietrich

Moira Dillon

Xuechen Ding

Ngoc Phuong (Phoebe) Dinh*

Wallace Dixon

Greta L. Doctoroff

Jessica Dollar

Sarah Domoff

Shannon Donofry

Rebecca Dore

Ann Dowker

Chantelle J. Dowsett

Natasha Duell

Adrienne Duke

Greg J. Duncan

Robert Duncan

Kristen Dunfield

Yarrow Dunham

William Dunlop

Myles Durkee

Stephanie Durrleman

Sebastian Dys

Sarah H. Eason

Mary B. Eberly Lewis

Leslie Echols

Peter Edelsbrunner

Maureen A. Eger

Franziska Ehrke

Katherine B. Ehrlich

Rina D. Eiden

Shiri Einav

Sierra Eisen*

Mona El-Sheikh

Kit Elam

Laura Elenbaas

Scott Engel

Jae Engle*

Jan Engelmann

M. Samantha English*

Elizabeth A. Enright

Stephen A. Erath

Christopher Erb

Cynthia A. Erdley

Ramirez Esparza

Graciela Espinosa-Hernandez

Guadalupe Espinoza

Alena G. Esposito
Idean Ettekal

Anthony Evans

E. Margaret Evans

Päivi Fadjukoff

Rachel H. Farr

Jo Ann M. Farver

Danielle Faulkner*

Roman Feiman

Xin Feng

Christopher T. Fennell

Brandee Feola

Gail Ferguson

Celeste Fernandez*

Diego Fernandez-Duque

Pier Ferrari

Laura Ferrer-Wreder

David E. Ferrier

Margaret A. Fields-Olivieri

Barbara H. Fiese

Courtney Filippi

Eric Daniel Finegood

Katherine Fiori

Anna V. Fisher

Paula Fite

Stanka A. Fitneva

Ryan Fitzgerald

Charles Fitzsimmons*

Robyn Fivush

Kaitlin M. Flannery

Chaia Flegenheimer*

Fenella Fleischmann

Jack M. Fletcher

Sammy Floyd

Sarah Foley

Tamsin Ford

R. Chris Fraley

Isabella Giulia Franzoi*

Doran French

Karin S. Frey

Ori Friedman

Margaret Friend

Christian Frings

Marina Fuertes

Andrew Fuligni

Bruce Fuller

David Funder

Maria Fusaro

Sarah E. Gaither

Colleen M. Ganley

Mengyu Miranda Gao

Claire F. Garandeau

Maria A. Gartstein

Ashley Gearhardt

Elizabeth T. Gershoff

Emily Gerstein

Deborah Giaschi

Stephen Gibson*

Matteo Giletta

Sarah Gillespie*

Ansley Tullos Gilpin

Jacquelyn Glidden*

Laura Glynn
Karrie Godwin

Adele E. Goldberg

Thalia R. Goldstein

Rebecca Gomez

Seçil Gönültaş

Antonya Gonzalez

David Gonzalez

Jackson Goodnight

Anthony Goodwin

Geoffrey Goodwin

Luc Goossens

Reyna Gordon

Benjamin Jacob Gorvine

Aner Govrin

Katharine Graf Estes

Sandra Graham

Susan Annetta Graham

Antonella Granieri

Josefina M. Grau

Ilaria Grazzani

Gustaf Gredeback

Mark Greenberg

Henriette Greischel

Kevin J. Grimm

Andrew Grogan-Kaylor

Ashley Groh

Julie Gros-Louis

Harold D. Grotevant

Chao $\mathrm{Gu}^{*}$

Nicole Guajardo

Fanny-Alexandra Guimond

Selin Gulgoz

Dominic Gullo

Elizabeth A. Gunderson

Megan R. Gunnar

Hanna Gustafsson

Amanda Guyer

Tilmann Habermas

Bat Sheva Hadad

Christine Hagerup*

Matthew Hagler*

Linda Halgunseth

John D. Haltigan

Kiley Hamlin

Stuart I. Hammond

Laura D. Hanish

Mary Hanley

Erin E. Hannon

Sara Harkness

Daniel Hart

Johanna Hartung

Naomi Hashimoto

Brenna Hassinger-Das

Paul D. Hastings

David Hawes

Skyler T. Hawk

Jessica Hay

Katherine Haydon

Marie J. Hayes

Karen Hazell Raine

Angela Xiaoxue He

Larisa Heiphetz

Nicole Heller* 
Kahl Hellmer

Andrea Helo

Charles Helwig

Kristi Hendrickson

Avishai Henik

Laura Hennefield

Jens Henrichs

Robert Hepach

Janette E. Herbers

Toria Herd*

Maciel M. Hernandez

Mikolaj Hernik

Todd I. Herrenkohl

Rachel Hershberg

Saeideh Heshmati

Thomas M. Hess

Gail D. Heyman

Leah Hibel

Liam J. B. Hill

Sarah E. Hill

James Benjamin Hinnant

Kathy Hirsh-Pasek

Aline Hitti

Justine Hoch

Darren Hocking

Claire Hofer

Erika Hoff

George W. Holden

Tom Hollenstein

Steven Holochwost

Shayla Holub

Claire Holvoet

Alice Sterling Honig

Stacey S. Horn

Caroline Byrd Hornburg

Camelia E. Hostinar

Carmel Houston-Price

Julie A. Hubbard

Matthew Hughes

Gizem Hülür

Alycia M. Hund

Carol Huntsinger

Yoon-Mi Hur

Hyesung Grace Hwang

Luke W. Hyde

\section{Elif Isbell}

Anne-Marie Iselin

Mitsuhiko Ishikawa

Jean M. Ispa

Todd Jackson

Robin Jacob

Lori James

Tim Janssen

Johanna Jarcho

Agnieszka Jaroslawska

Chris Jarrold

Vikram K. Jaswal

Sarah Jessen

Yuhong Jiang

Jamie Jirout

Rosanne M. Jocson

Elizabeth Johnson
Matthew D. Johnson

Sara K. Johnson

Angie Johnston

Damon Jones

Lauren Jones*

Shawn Jones

Nancy C. Jordan

John T. Jost

Joshua Juvrud

George Kachergis

Girija Kadlaskar

Marina Kalashnikova

Priya Kalra

Goda Kaniušonytë

Jeremy Kanter

Sabina Kapetanovic

Peter Kaplan

Lana Karasik

Juliana Karras-Jean Gilles

Joscha Kärtner

Laura Katus

Fumito Kawakami

Loes Keijsers

Heidi Keller

David J. Kelly

Ryan J. Kelly

Charles Kemp

Ben Kenward

Kathryn A. Kerns

Gizem Keskin*

Zainab Khan*

Jennifer Khoury

Rogier Kievit

Ryan Kilmer

JaHun Kim

Sunae Kim

Young-Suk Grace Kim

Jungmeen Kim-Spoon

Bijan Kimiagar

Sarah Kiperman

Heather Kirkorian

Patricia Kitsao-Wekulo

Daniel N. Klein

Wendy Kliewer

André Knops

Grazyna Kochanska

Amanda Koehn

Tehila Kogut

Jonathan F. Kominsky

Tanja Könen

Tuire Koponen

Reza Kormi-Nouri

Moritz Köster

Yulia Kovas

Haley E. Kragness

Hannah J. Kramer*

Saloni Krishnan

Lorraine Kubicek

Yana Kuchirko

Janet E. Kuebli

Valerie Kuhlmeier

Deanna Kuhn

Beth Kurtz-Costes
Tamar Kushnir

Sierra Kuzava

Lia Kvavilashvili

Jin Kyong-sun

Jennifer C. LaBounty

Gary W. Ladd

Kristin H. Lagattuta

Catherine Laing

Robert D. Laird

Sharon F. Lambert

Steven Landau

Karin Landerl

Susan H. Landry

Michael Langlais

Kimberly Langrehr

Ann Lantagne

Jill Lany

Elizabeth Lapidow*

Reed Larson

Jillian Lauer

Heidemarie K. Laurent

Sean Laurent

John Lavigne

Josh Lawrence

Laurel Lawyer

Campbell Leaper

Michele Lease

Joshua Lee

Julie Lee

Tae Kyoung Lee

Esther Leerkes

Jo-Anne LeFevre

Jordan Legaspi*

Ryan Lei

Erin Leichman

Maria Lent*

Jacqueline V. Lerner

Arne Lervåg

Leah M. Lessard

Shiri Lev-Ari

Jacqueline Leventon

Dani Levine

Joerg Lewald

$\mathrm{Gu} \mathrm{Li}$

Pearl Han Li

Yan Li

Melissa Libertus

Diane Lickenbrock

Jeffrey Liew

Angeline S. Lillard

Betty Lin

Thomson J. Ling

Maria Beatriz Linhares

Karina J. Linnell

Francesca Lionetti

Melissa A. Lippold

Shiyao Liu*

Shizhu Liu*

Yangyang Liu*

Kimberly M. Livingstone

Karin Loh

Elisabetta Lombardi

Caitlin McPherran Lombardi
Christopher J. Lonigan

Lisa Lopez

Hugues Lortie-Forgues

Jessica P. Lougheed

Stella Lourenco

Tom Lowrie

Fantasy Taina Lozada

Linda Luecken

Priscilla Lui

Thomas D. Lyon

Ashley Lytle

Weiyi Ma

Cyrille Magne

Natasha Magson

Anne J. Maheux*

Tristan Mahr

Caitlin E. V. Mahy

Alexandra Main

Steve Majerus

Hio Wa Mak

Sarah T. Malamut

Margherita Malanchini

Jeannette Mancilla-Martínez

Tara Mandalaywala

Frank Mann

Catherine Manning

Josita Maouene

Kristine Marceau

Vesna Marinovic

Charlotte Markey

Hanna Marno

Elizabeth Marsh

Herbert W. Marsh

Peter Marshall

Carol L. Martin

Gema Martin-Ordas

Olivier Mascaro

Clay Mash

Shira Mattera

Danielle Matthews

Jamaal Sharif Matthews

John Maule

Emily M. May

Katherine McAuliffe

Allyssa McCabe

Teresa McCormack

Meghan McCormick

Kristina McDonald

Meredith McGinley

Luke McGuire

Susan M. McHale

Clark McKown

Niamh McLoughlin

Sinead McNally

Nicole M. McNeil

Adam Meade

Alan Meca

Michael Medina

Wim Meeus

Elizabeth Meins

Samuel Meisel

Monica Melby-Lervåg

Gabriela Memba* 
Ersilia Menesini

Marek Meristo

Rebecca Merkley

Christine E. Merrilees

Emily Messer

Diana Meter

Aaron Metzger

Kalina J. Michalska

George F. Michel

Amori Yee Mikami

Cindy Miller

Hilary Miller

Jonas G. Miller

Stephanie E. Miller

Laura Miller-Graff

William Roger Mills-Koonce

Luke Miratrix

Sheena Mirpuri

Rashmita S. Mistry

Kelly S. Mix

Kathryn Lynn Modecki

Kameron Moding

Dylan Molenaar

Catherine Molho

Henrike Moll

Monika Molnar

Catherine Mondloch

Claire Monroy

Ginger A. Moore

Santiago Morales

Diana Morelen

Candice Morey

Elizabeth Morgan

Paul L. Morgan

Elizabeth Morin-Lessard

Gwynn Morris

J. Bruce Morton

Ughetta Moscardino

Louis J. Moses

Margaret Moulson

Nina S. Mounts

Christoph Mueller

Emma Mumper*

Francesca Muntoni

Desiree Murray

Lauren Mutignani*

Sarah Myruski

Ane Nærde

Shaylene Nancekivell

Misaki N. Natsuaki

Elena Nava

Amy Needham

David A. Nelson

Eliza L. Nelson

Timothy D. Nelson

Dezso Nemeth

Kimberly Turner Nesbitt

Jacqueline Nesi

Andreas B. Neubauer

Florrie Fei-Yin Ng

Jana Nikitin

Adrienne Nishina

Nicholaus S. Noles
Saara Nolvi

Iris Nomikou

Erik Nook*

Alexander Noyes*

Larry Nucci

Amy K. Nuttall

Angela Nyhout

Rosalie Odean

Noa Ofen

Yoonkyung $\mathrm{Oh}$

Kristina Olson

Sheryl Olson

Desmond Ong

John Opfer

David Oppenheim

Peter A. Ornstein

Assaf Oshri

Francois Osiuriak

Ori Ossmy

Christopher Osterhaus

Brendan Dale Ostlund

Jamie M. Ostrov

Ora Oudgenoeg-Paz

Harriet Over

Daniel Ozer

Jenny Padilla

Diego Palacios

Carolyn Palmquist

Carlomagno Panlilio

Ye Rang Park*

Lindsey Partington*

Concetta Pastorelli

Monisha Pasupathi

Meagan Patterson

James Paulson

Ayse Payir

Alvin Pearman

Margot Peeters

Kätlin Peets

Santiago Pelegrina

Peng Peng

Marie-Hélène Pennestri

Rebecca Peretz-Lange

Norma Perez-Brena

David G. Perry

Nicole B. Perry

Sarah Perzow

Carole Peterson

Stephen (Steve) Petrill

Yaacov Petscher

Jenny Phan

Lauren Philbrook

Robert C. Pianta

Bradford H. Pillow

Andres Pinedo*

Martin Pinquart

Tiago Miguel Pinto

Alex Piquero

Elizabeth Planalp

Michael Pluess

Courtney Pollack

Seth Pollak*
Kristie Poole*

V. Paul Poteat

Diane Poulin-Dubois

Nausicaa Pouscoulous

J. Loes Pouwels

Lindsey Powell

Laurent Pretot

Heather L. Price

Shannon Pruden

Jennie E. Pyers

Yang Qu

Jon Quach

Claudia Quaiser-Pohl

Hugh Rabagliati

K. Lee Raby

Eva Rafetseder

Marcela Raffaelli

Qazi Rahman

David H. Rakison

Hannes Rakoczy

Lakshmi Raman

Shana Ratcliff*

Catherine Ratelle

James Vance Ray

Kirsten L. Read

Rachael D. Reavis

Holly Recchia

Jonathan Redshaw

Elaine Reese

Leslie Rescorla

Bolivar Reyes Jaquez

Valerie Reyna

Bert Reynvoet

Mijke Rhemtulla

Jean E. Rhodes

Stephen Rhodes

Andrew Ribner

Matthew Richardson*

Julia Braungart Rieker

Elizabeth M. Riina

Luke Rinne

Camille Rioux

Charlie Rioux

Paolo Riva

Christina Riva Crugnola

Michael T. Rizzo

Savannah R. Roberts*

Steven O. Roberts

Vanessa Rodriguez*

Barbara Rogoff

Daniel Romer

Anna-Sophie Rommel

Samuel Ronfard

Katie Rosanbalm

Amanda J. Rose

David Rosenfield

Johanna Rosenqvist

Isabelle Roskam

Josephine Ross

Federico Rossano

Sharon Rostosky

William Andrew Rothenberg
Joshua Rottman

Paula Rubio-Fernandez

David Rudek

Mark Runco

Helena Rutherford

Allison Ryan

Rebecca M. Ryan

Abraham Sagi-Schwartz

Farzana Saleem

Virginia Salo

Adriana Sampaio

Valerie San Juan

Carol Sansone

Barbara W. Sarnecka

Kimberly J. Saudino

Marissa Sbrilli*

Maja Schachner

Hannah Lindsay Schacter

Katherine Schaumberg

Suzy Scherf

Alice Schermerhorn

Louis A. Schmidt

Sara A. Schmitt

David Schultz

Wiebke Schulz

David Schwartz

Rebecca Schwartz-Mette

Jason Scofield

Katharine Scott*

Rose Scott

Nuria Sebastian Galles

Amanda Seed

Diana Selmeczy

Sawa Senzaki

Holly Shablack

Ameneh Shahaeian

Neika Sharifian

Alex Shaw

Daniel S. Shaw

Madelynn Shell

Mark Sheskin

Grant S. Shields

Elizabeth Shirtcliff

Mina Shimizu*

Priya Mariana Shimpi

Rebecca Shiner

Kenneth Shores

Elizabeth P. Shulman

Kristin Shutts

Luigia Simona Sica

Jellie Sierksma

Cynthia Siew

Jelle J. Sijtsema

Jennifer Susan Silk

Cassandra Simmel

Nina Simms

Victoria Simms

Sandra Simpkins

Leher Singh

Sylvain Sirois

Alice Skelton

Ewa Skimina

Allison Skinner 
Cynthia L. Smith Emilie Phillips Smith

Kevin Smith

Rhiannon L. Smith

Mia Smith-Bynum

Catherine E. Snow

Sara Sohr-Preston

Gaye Soley

Leah H Somerville

Savannah Sommers*

Hyun-Joo Song

Bailey Speck*

Matthew G. Springer

Angela D. Staples

Håkan Stattin

Ursula M. Staudinger

Christine Steeger

Jennifer Steele

Laurence Steinberg

Ryan Stevenson

Sunita M. Stewart

Jessica Stoltzfus Grady

Tawni Stoop*

Hannah Strauß

Melissa Sturge-Apple

Catalina Suarez-Rivera

Kazumi Sugimura

Xiaoran Sun

Charles Super

Cynthia Suveg

H. Lee Swanson

Johnna Swartz

Daniel Swingley

Moin Syed

Emily Szkudlarek

Christine Tai*

Andrea Takahesu Tabori

Nicole M. Talge

Victoria Talwar

Catherine S. Tamis-LeMonda

Vivian Tamkin*

Teresa Tang*

Amanda Tarullo

Nasim Tavassoli*

Lorraine C. Taylor

Brandon F. Terrizzi

Michael Henry Tessler

Douglas M. Teti
Rachel M. Theodore

William Therrien

Rachel Thibodeau

Jochem Thijs

Robert Thornberg

Sabrina L. Thurman

Jing Tian

Liat Tikotzky

James Torrence Todd

Elena Toffol

Przemyslaw Tomalski

Michael Tomasello

Carolyn Tompsett

Vasiliki Totsika

Raphael Travis

Rebecca Treiman

Sam Trejo

Kim M. Tsai

Monica Tsethlikai

Lily Tsoi

Kelly M. Tu

Corinna Tucker

Jonathan Tudge

Eric Turkheimer

Katherine E. Twomey

Kimberly Tyler

Kathryn Unruh

Alexandra Ursache

Miguel Vadillo

Tracy Vaillancourt

Uku Vainik

Amrisha Vaish

Carlos Valiente

Patti M. Valkenburg

Riet van Bork

Daphne van de Bongardt

Alithe Van den Akker

Marion van den Heuvel

Jolien van der Graaff

Jean Baptiste Van der Henst

Lotte van Doeselaar

Martijn Van Heel

Angeliek van Hout

Carol Van Hulle

Deborah Lowe Vandell

Maarten Vansteenkiste

Sara A. Vasilenko
Nadya Vasilyeva

Brian E. Vaughn

Lauren Vazquez*

Alexander T. Vazsonyi

Luis Vega

Harriet J. Vermeer

Marilyn Vihman

Adam Voight

Anthony Volk

Justin William Vollet

Brenda L. Volling

Alexander von Eye

Maria von Salisch

Elizabeth Votruba-Drzal

Martha E. Wadsworth

Jenny Wagner

Laura Wagner

Jeffrey J. Walczyk

Caren M. Walker

Eric A. Walle

Joy Wang

Jun Wang

Meifang Wang

Qian Wang

Su-Hua Wang

Wen Wang*

Yijie Wang

Wayne Warburton

Julie Wargo Aikins

Felix Warneken

Katherine Warnell

Sam Wass

Shoko Watanabe

Everett Waters

Sara F. Waters

Rod J. Watts

Drew Weatherhead

Molly S. Weeks

Adriana Weisleder

Alexander Weiss

Michael Weiss

Janet A. Welsh

Nicole Wen

Eric Wesselmann

Kelsey West

Bridget Weymouth

Diana Whalen

Margaret Whedon
Katherine White

Rebecca M. B. White

Sherri Widen

Sandra A. Wiebe

Margit F. Wiesner

Allan Wigfield

Eric Wilkey

Matti Wilks

Amanda Williams

Kate Williams

Ariel Williamson

Cynthia Willis Esqueda

Julia Wilson*

Liliane Windsor

Adam Winsler

Catherine Winsper

David C. Witherington

Erica Hsiung Wojcik

Sharon Wolf

Steven Woltering

Vivian Wong

Mary Woody

Yang Wu

Naiqi G. Xiao

Natalie Xiong ${ }^{*}$

Jing $\mathrm{Xu}$

Yiyuan Xu

Fan Yang

Yang Yang

Jing $\mathrm{Yu}$

Yue Yu

Lei Yuan

Kristen Yule*

Henrik Zachrisson

Imac Maria Zambrana

Lynne Zarbatany

Nicole Zarrett

Philip D. Zelazo

Marcel Zentner*

Megan Zeringue*

Selin Zeytinoglu

Xiao Zhang

Susannah Zietz

Peter Zimmermann

Antonio Zuffiano

Jennifer Zuk

* Denotes a reviewer who co-reviewed under the supervision of a primary reviewer. 